Rosângela Ghiringhelli1

Maria Cecília Martinelli lorio²

resumo

Objetivo: Verificar os efeitos da estimulação acústica por meio do uso de próteses auditivas em idosos com avaliação sugestiva de alteração cognitiva. Método: Foram avaliados 31 idosos com perda auditiva neurossensorial bilateral simétrica de grau até moderadamente severo com escore sugestivo de alteração cognitiva, sendo 15 do gênero masculino e 16 do feminino com idades de 60 a 80 anos. Estes idosos foram atendidos no serviço de Saúde Auditiva do Hospital Universitário da Escola Paulista de Medicina/UNIFESP e (re) avaliados por meio do teste Alzheimer's Disease Assessment Scalecognitive subscale (ADAS-Cog) aplicado antes, quatro e oito meses após a adaptação de próteses auditivas. A adaptação das próteses auditivas foi realizada conforme o preconizado pela política Nacional de Atenção à Saúde Auditiva do Governo Federal - Brasil (Portaria

1 Graduada em Fonoaudiologia. Mestre em Ciências (Distúrbios da Comunicação Humana: Campo Fonoaudiológico) pela Escola Paulista de Medicina da UNIFESP. E-mail: fga.rosangela@yahoo.com.br. 2 Graduada em Fonoaudiologia. Professora-Associada e Livre Docente do Departamento de Fonoaudiologia da Escola Paulista de Medicina da UNIFESP. E-mail: cmartinelli@uol.com.br. 
SAS/MS N 432, de 14/11/2000). A análise de dados foi realizada por meio de estatística descritiva, teste de Análise de Variância (ANOVA) e comparações múltiplas de Tukey com nível de significância de 5\%. Resultados: As 11 tarefas do ADAS-Cog foram agrupadas em três domínios cognitivos: memória, praxia e linguagem. O estudo mostrou que houve uma redução gradativa significante $(p<0,001)$ nos escores ao longo das avaliações, denotando melhora nas respostas ao teste. O percentual de idosos com resultados sugestivos de alteração cognitiva passou de 100\% (etapa pré-intervenção) para 41,9\% e 22,6\% respectivamente quatro e oito meses após a adaptação das próteses auditivas. Conclusão: Os idosos apresentaram melhora nas respostas ao teste ADAS-Cog após a estimulação acústica por meio do uso de próteses auditivas.

palavras-chave

Auxiliares de Audição. Questionário. Cognição. Idosos.

\section{Introdução}

O envelhecimento da população é um efeito mundial, caracterizado por um período de declínio físico, psíquico, social e mental do indivíduo e apresenta consequências diretas nos sistemas de saúde pública (RUSSO, 1999; BRASIL, 2000; BARRETO, 2006). O Brasil também acompanha este fenômeno (IBGE, 2010) e associado ao envelhecimento surgem algumas co-morbidades. Dentre elas, tem-se a perda auditiva decorrente do envelhecimento ou presbiacusia, que é caracterizada por um declínio da função auditiva, uma deterioração progressiva das células ciliadas com consequente redução da sensibilidade auditiva, em especial em altas frequências (KIESSLING et al., 2003).

A perda auditiva, em função da perda de audibilidade (menor acesso aos sons de fala), causa limitações e restrições nas atividades de vida diária, o que pode favorecer o isolamento social e intelectual, eventuais distúrbios psiquiátricos e piora da qualidade de vida (RUSSO et al., 2003; MARQUES et al., 2004; GATES; MILLS, 2005).

Alguns autores consideram que a deterioração da função auditiva é uma das mais graves deficiências presentes no envelhecimento (SANTIAGO; NOVAES, 2009). 
Também presente no envelhecimento há o declínio cognitivo com a degradação dos processos cognitivos como memória, linguagem, habilidades construtivas entre outros, que prejudicam as operações atencionais e de controle executivo (ARNETT et al., 1997). As alterações de memória constituem prejuízo importante (LIGHT; SINGH, 1987). Especificamente, tem-se observado que a perda auditiva impacta negativamente a memoria operacional que é importante para a compreensão de fala especialmente em situações de escuta difícil como, por exemplo, na presença de ruído (DOHERTY; DESJARDINS, 2015).

Hipóteses definidas pelo grupo de Estudo de Berlin sobre o Envelhecimento - do inglês Berlin Aging Study - BASE, tentam explicar a relação entre percepção auditiva e cognição no envelhecimento. Os pesquisadores do BASE concluíram que a interação entre processamento auditivo e cognitivo em idosos com perda auditiva, resulta em maior esforço para escutar, limitando o uso de processos mentais disponíveis para compreender e recordar o que foi ouvido (BALTES; LINDENBERG, 1997). Observaram também que há diferenças individuais relacionadas também com a idade, no reconhecimento de fala em situação de escuta difícil (relação Sinal/Ruído - S/R) no qual existe extremo esforço para escutar, podendo ser minimizado simplesmente ao ajustar esta relação. A partir destes achados e considerando a hipótese de que o declínio perceptual e a entrada perceptual pobre resultam em reduzido desempenho cognitivo Pichora-Fuller (2006) sugeriu como intervenção a reabilitação audiológica e relatou as implicações importantes nesta reabilitação. Uma delas é que além da alteração auditiva relacionada com o envelhecimento, diferenças na utilização dos recursos cognitivos limitados para escutar podem restringir a participação plena desses indivíduos em atividades de vida diária que exijam escuta, compreensão e comunicação. A outra é que a audibilidade pode ser parcial ou totalmente restaurada com a intervenção por meio da adaptação de próteses auditivas, o que resulta não somente em melhor percepção de fala, mas também em melhor função cognitiva em termos de habilidade para compreender e recordar o que foi ouvido.

Há evidências que o uso de próteses auditivas pode melhorar o desempenho de idosos em testes apresentados verbalmente porque o sinal amplificado permite a melhora da percepção das instruções e dos itens do teste (MULROW et al., 1990; ALLEN et al., 2003). O estudo de Weinstein e Amsel (1986) verificou que em um grupo de idosos com demência, estes foram reclassificados em categorias menos severas de demência quando avaliados utilizando amplificação.

Um teste amplamente usado para avaliar os processos cognitivos é o ADAS-Cog (Alzheimer's Disease Assessment Scale-Cognitive subscale). Esse teste foi traduzido e validado para sua utilização no Brasil (SCHULTZ; SIVIERO; 
BERTOLUCCI, 2001). É considerado o "padrão-ouro" em estudos de intervenção para a avaliação do status cognitivo. Sofre importante influência da escolaridade e avalia principalmente os domínios cognitivos da memória, da linguagem e das praxias. Como a cognição também pode ser afetada pelas doenças cerebrais degenerativas, esses domínios cognitivos merecem especial atenção.

Com base no exposto acima, este estudo teve como objetivo verificar os efeitos da estimulação acústica por meio do uso de próteses auditivas no desempenho cognitivo de idosos com avaliação sugestiva de alteração cognitiva.

\section{Método}

Esta pesquisa foi realizada no Núcleo Integrado de Assistência, Ensino e Pesquisa em Audição - NIAPEA (EPM/UNIFESP/Brasil) durante os anos de 2011 e 2012, após aprovação do Comitê de Ética e Pesquisa desta Instituição. Constou de um estudo clínico longitudinal que avaliou e comparou o desempenho cognitivo de idosos, novos usuários de próteses auditivas quanto aos domínios relativos à memória, linguagem e praxia. Inicialmente, todos os indivíduos consentiram a sua participação no estudo mediante a assinatura do Termo de Consentimento Livre e Esclarecido.

Para seleção da amostra, os seguintes critérios de elegibilidade foram estabelecidos: ter idade igual ou superior a 60 anos; ser portador de perda auditiva neurossensorial de grau leve a moderadamente severo bilateral (média dos limiares de audição de 26 a 70 dBNA nas frequências de 500, 1000 e 2000 Hz) (DAVIS; SILVERMAN, 1970); apresentar resultado sugestivo de alteração cognitiva por meio do teste Alzheimer's Disease Assessment Scale - cognitive subscale (ADAS_Cog) e não ser usuário de próteses auditivas, anteriormente ao início desta pesquisa.

A avaliação constou da aplicação do teste Alzheimer's Disease Assessment Scale - Cognitive Subscale - ADAS-Cog antes da adaptação de próteses auditivas. A seguir, foram adaptadas as próteses auditivas de tecnologia digital com amplificação não linear, dispensadas pelo Sistema Único de Saúde SUS, segundo a Política Nacional de Atenção à Saúde Auditiva do Governo Federal - Brasil (Portaria SAS/MS N 432, de 14 de novembro de 2000).

Os participantes após a adaptação foram acompanhados por um mês para orientações e ajustes das regulagens das próteses auditivas se necessário, a fim de garantir o uso efetivo das mesmas. Todos os participantes foram reavaliados quatro e oito meses após o início do uso da prótese auditiva por meio do teste ADAS-Cog. 
A Escala de Avaliação da Doença de Alzheimer (ADAS) foi desenvolvida com o intuito de avaliar a cognição e as mudanças não cognitivas, que são características dessa doença. Esta escala é composta por duas partes com uma pontuação máxima de 120 pontos. Uma delas, utilizada neste estudo, foi o ADAS-Cog, medida da função cognitiva baseada no desempenho do sujeito avaliado. O ADAS-Cog, composto por 11 itens, avalia memória, linguagem, praxia e compreensão de comandos. A pontuação máxima de toda a avaliação cognitiva é de 70 pontos e quanto maior a pontuação, maior o comprometimento cognitivo do indivíduo. Considerando-se a escolaridade, será declarado indivíduo sugestivo de alterações cognitivas aquele que apresentar: escore acima de 23,3 para até quatro anos de escolaridade; acima de 13,4, de cinco a 11 anos de escolaridade; e escore acima de 11,1, de 12 ou mais anos de escolaridade (BERTOLUCCI et al., 2001). Este teste foi aplicado pela pesquisadora, com duração de 35 a 45 minutos.

Após a coleta dos dados, optou-se por agrupar as 11 tarefas do teste ADAS-Cog em três domínios: memória - palavra evocada, orientação, reconhecimento de palavras e recordação/lembrança; linguagem - nomeação de objetos e dedos, comandos, linguagem falada, dificuldade na fala espontânea e compreensão; praxia - praxias construtiva e ideativa. Nesta situação de agrupamento e considerando a média etária do grupo, têm-se os seguintes escores para considerar o indivíduo sem alterações cognitivas por domínio: memória - até 7,5 pontos, linguagem - até 1,6 pontos e praxia - até 1,8 pontos.

A amostra foi caracterizada quanto a gênero, idade e escolaridade, por meio de estatística descritiva.

Para o comparativo entre as etapas (doação, 4 meses e 8 meses) para os domínios de memória, linguagem e praxia, foi aplicado o teste de Análise de Variância (ANOVA) com nível de significância de 5\%. Se detectada diferença, realizou-se o teste Comparações Múltiplas de Tukey para identificar quais as diferenças significantes. Em todos os testes, foi fixado nível de significância de 0,05 .

\section{Resultados}

A seguir, são apresentados os resultados obtidos na avaliação de 31 idosos, de 60 a 80 anos de idade, novos usuários de próteses auditivas, sendo $16(51,6 \%)$ do gênero masculino e $15(48,4 \%)$ do feminino. A média etária do grupo foi de 70,6 anos. A Tabela 1 apresenta estatísticas descritivas para a idade, em anos, da amostra. 
Tabela 1 - Estatísticas descritivas para a idade (anos) da amostra.

\begin{tabular}{c|c|c|c|c|c}
\hline $\mathbf{n}$ & Média & $\begin{array}{c}\text { Desvio } \\
\text { padrão }\end{array}$ & Mínimo & Mediana & Máximo \\
\hline 31 & 70,6 & 5,9 & 60 & 69,5 & 80 \\
\hline
\end{tabular}

Legenda: $\mathrm{n}$ = número de idosos no grupo.

Fonte: Tabela elaborada pelas autoras.

Valores de estatísticas descritivas para a escolaridade (em anos) da amostra são apresentados na Tabela 2.

Tabela 2 - Estatísticas descritivas para a escolaridade (anos) da amostra.

\begin{tabular}{c|c|c|c|c|c}
\hline $\mathbf{n}$ & Média & $\begin{array}{c}\text { Desvio } \\
\text { padrão }\end{array}$ & Mínimo & Mediana & Máximo \\
\hline 31 & 4,9 & 3,5 & 0 & 4 & 12 \\
\hline
\end{tabular}

Legenda: $n$ = número de idosos no grupo.

Fonte: Tabela elaborada pelas autoras.

A Tabela 3 apresenta o comparativo entre as etapas (Doação, quatro e oito meses) para o domínio de Memória. Para tanto foi aplicado o teste de Análise de Variância (ANOVA). Se detectada diferença realizou-se o teste Comparações Múltiplas de Tukey (Tabela 4).

Tabela 3 - Estatística descritiva para o domínio Memória nas etapas de (re)avaliação.

\begin{tabular}{l|c|c|c}
\hline & Doação & 04 meses & 08 meses \\
\hline Média & 11,56 & 9,67 & 8,06 \\
\hline Desvio-padrão & 3,40 & 4,24 & 2,99 \\
\hline $\mathrm{N}$ & 31 & 31 & 31 \\
\hline
\end{tabular}

Legenda: $n$ = número de idosos no grupo.

Fonte: Tabela elaborada pelas autoras.

Doação = início do uso de próteses auditivas não lineares.

04 meses = 04 meses de uso de próteses auditivas não lineares.

08 meses $=08$ meses de uso de próteses auditivas não lineares.

ANOVA (p) $<0,001^{*}$ 
Tabela 4 - Resultado do Teste de Comparações múltiplas de Tukey para o domínio Memória.

\begin{tabular}{c|c|c|c}
\hline & Doação & 04 meses & 08 meses \\
\hline Doação & & $0,003^{*}$ & $<0,001^{*}$ \\
\hline 04 meses & $0,003^{*}$ & & $0,007^{*}$ \\
\hline 08 meses & $<0,001^{*}$ & $0,007^{*}$ & \\
\hline
\end{tabular}

Legenda: $\mathrm{n}$ = número de idosos no grupo.

Fonte: Tabela elaborada pelas autoras.

Doação = início do uso de próteses auditivas não lineares.

04 meses = 04 meses de uso de próteses auditivas não lineares.

08 meses $=08$ meses de uso de próteses auditivas não lineares.

$\left(^{\star}\right)=$ resultado estatisticamente significante

Analisando os dados apresentados nas Tabelas 3 e 4 conclui-se que houve diferença significante $(p=0,003)$ entre as etapas doação e quatro meses de uso de próteses auditivas e entre as etapas doação e oito meses de uso das mesmas $(\mathrm{p}<0,001)$, mostrando um declínio na pontuação média entre as etapas de (re) avaliação e consequentemente melhora da memória. A Figura 1 apresenta o gráfico descritivo do domínio memória para melhor visualização dos resultados.

Figura 1 - Gráfico descritivo para o domínio memória nas etapas de (re)avaliação.

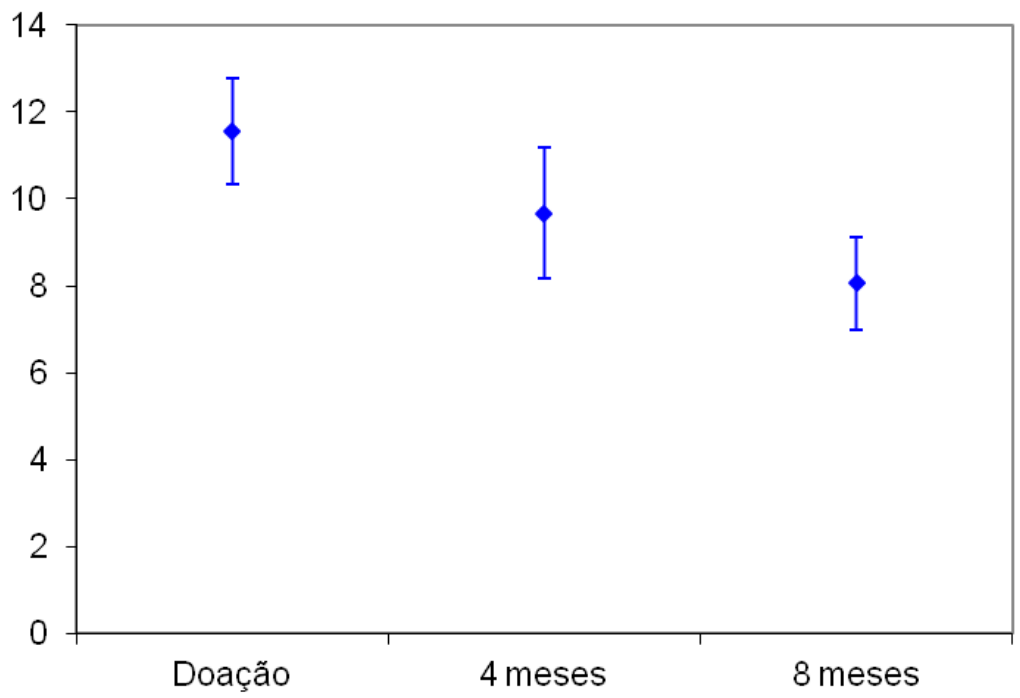

Legenda: Intervalo de confiança para a média: média \pm 1,96 * desvio-padrão / $\sqrt{ }(\mathrm{n}-1)$.

Fonte: Figura elaborada pelas autoras. 
A Tabela 5 apresenta o comparativo entre as etapas (Doação, quatro e oito meses) para o domínio de Linguagem. Para tanto foi aplicado o teste de Análise de Variância (ANOVA). Se detectada diferença realizou-se o teste Comparações Múltiplas de Tukey (Tabela 6).

Tabela 5 - Estatística descritiva para o domínio Linguagem nas etapas de (re)avaliação.

\begin{tabular}{c|c|c|c}
\hline & Doação & 04 meses & 08 meses \\
\hline Média & 6,76 & 6,18 & 5,26 \\
\hline Desvio-padrão & 1,66 & 1,66 & 2,00 \\
\hline $\mathrm{N}$ & 31 & 31 & 31 \\
\hline
\end{tabular}

Legenda: $n$ = número de idosos no grupo.

Doação = início do uso de próteses auditivas não lineares.

04 meses $=04$ meses de uso de próteses auditivas não lineares.

08 meses $=08$ meses de uso de próteses auditivas não lineares.

ANOVA $(p)<0,001^{*}$

Fonte: Tabela elaborada pelas autoras.

Tabela 6 - Resultado do Teste de Comparações múltiplas de Tukey, para o domínio Linguagem.

\begin{tabular}{c|c|c|c}
\hline & Doação & 04 meses & 08 meses \\
\hline Doação & & 0,098 & $<0,001^{*}$ \\
\hline 04 meses & 0,098 & & $0,009^{*}$ \\
\hline 08 meses & $<0,001^{*}$ & $0,009^{*}$ & \\
\hline
\end{tabular}

Legenda: $n$ = número de idosos no grupo.

Fonte: Tabela elaborada pelas autoras.

Doação = início do uso de próteses auditivas não lineares.

04 meses = 04 meses de uso de próteses auditivas não lineares.

08 meses $=08$ meses de uso de próteses auditivas não lineares.

$\left(^{*}\right)=$ resultado estatisticamente significante

Analisando os dados apresentados nas Tabelas 5 e 6 conclui-se que houve diferença significante entre as etapas de doação e oito meses de uso de próteses auditivas $(\mathrm{p}<0,001)$. $\mathrm{O}$ mesmo ocorreu entre as etapas de quatro e oito meses pós-doação $(p=0,009)$, evidenciando melhora no desempenho dos idosos quanto ao domínio linguagem com o tempo de uso de seus dispositivos de amplificação sonora. A Figura 2 apresenta o gráfico descritivo do domínio de Linguagem para melhor visualização dos resultados. 
Figura 2 - Gráfico descritivo para o domínio linguagem nas etapas de (re)avaliação.

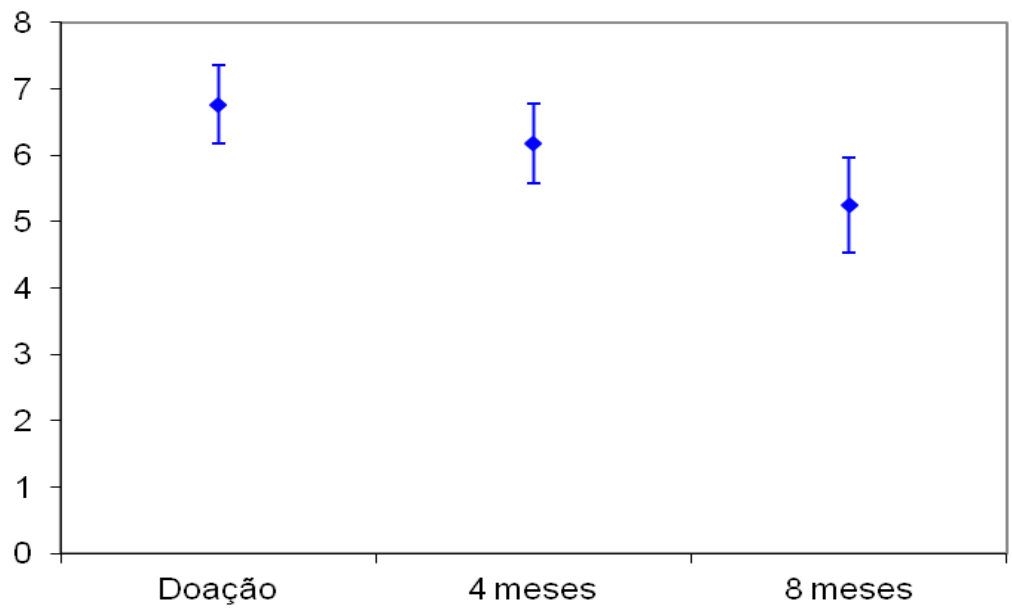

Legenda: Intervalo de confiança para a média: média \pm 1,96 * desvio-padrão / $\sqrt{ }(\mathrm{n}-1)$.

Fonte: Figura elaborada pelas autoras.

A Tabela 7 apresenta o comparativo entre as etapas (doação, quatro e oito meses) para o domínio de Praxia. Para tanto foi aplicado o teste de Análise de Variância (ANOVA). Se detectada diferença realizou-se o teste Comparações Múltiplas de Tukey (Tabela 8).

Tabela 7 - Estatística descritiva para o domínio Praxia nas etapas de (re)avaliação.

\begin{tabular}{c|c|c|c}
\hline & Doação & 04 meses & 08 meses \\
\hline Média & 3,32 & 2,71 & 2,32 \\
\hline Desvio-padrão & 1,51 & 1,75 & 1,42 \\
\hline $\mathbf{N}$ & 31 & 31 & 31 \\
\hline
\end{tabular}

Legenda: $n$ = número de idosos no grupo.

Doação = início do uso de próteses auditivas não lineares.

04 meses = 04 meses de uso de próteses auditivas não lineares.

08 meses $=08$ meses de uso de próteses auditivas não lineares.

ANOVA $(p)<0,001^{*}$

Fonte: Tabela elaborada pelas autoras. 
Tabela 8 - Resultado do Teste de Comparações múltiplas de Tukey para o domínio Praxia.

\begin{tabular}{c|c|c|c}
\hline & Doação & 04 meses & 08 meses \\
\hline Doação & & $0,021^{*}$ & $<0,001^{*}$ \\
\hline 04 meses & $0,021^{*}$ & & 0,070 \\
\hline 08 meses & $<0,001^{*}$ & 0,070 & \\
\hline
\end{tabular}

Legenda: $n$ = número de idosos no grupo.

Doação = início do uso de próteses auditivas não lineares.

04 meses = 04 meses de uso de próteses auditivas não lineares.

08 meses $=08$ meses de uso de próteses auditivas não lineares.

$\left({ }^{*}\right)=$ resultado estatisticamente significante

Fonte: Tabela elaborada pelas autoras.

Analisando os resultados apresentados nas Tabelas 3 e 4 conclui-se que houve diferença estatisticamente significante entre as etapas doação e quatro meses de uso de próteses auditivas $(\mathrm{p}=0,021)$ e entre as etapas de doação e oito meses pós-adaptação $(\mathrm{p}<0,001)$ comprovando a melhora dos idosos neste domínio. A Figura 3 apresenta o gráfico descritivo do domínio de Praxia para melhor visualização dos resultados.

Figura 3 - Gráfico descritivo para o domínio praxia nas etapas de (re)avaliação.

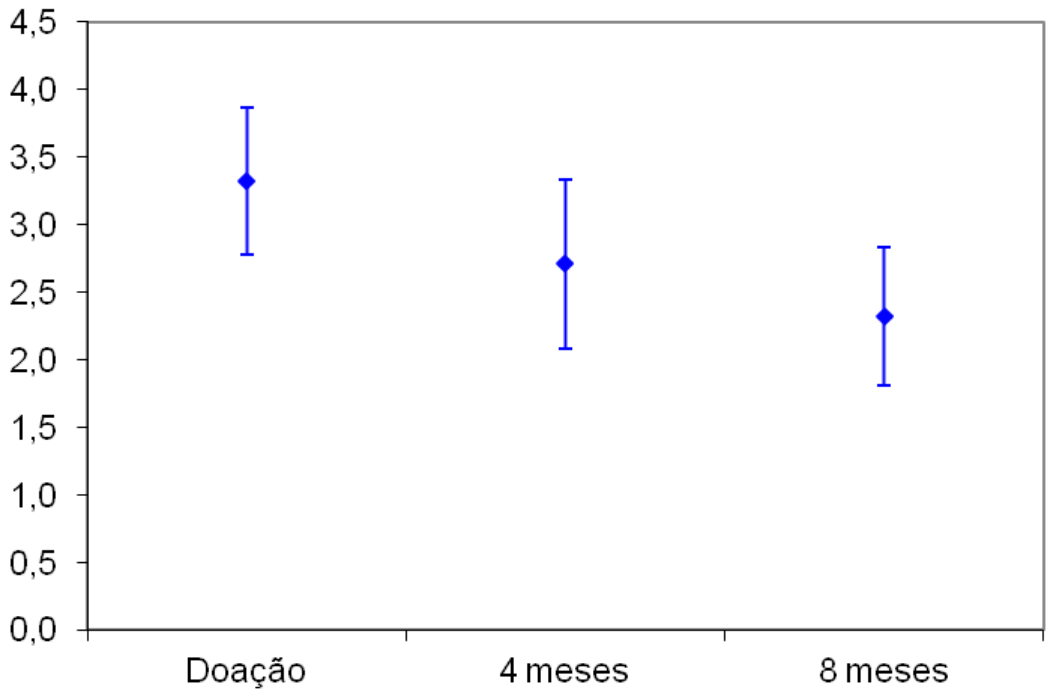

Legenda: Intervalo de confiança para a média: média \pm 1,96 * desvio-padrão / $\sqrt{ }(\mathrm{n}-1)$. Fonte: Figura elaborada pelas autoras. 
Na Tabela 9 são apresentadas as distribuições de frequências e porcentagens do ADAS-Cog categorizado em normal e alterado, nas etapas pré-estimulação auditiva, quatro e oito meses após a doação de próteses auditivas não lineares.

Tabela 9 - Distribuições de frequências e porcentagens do ADAS-Cog categorizado (Normal x Alterado) nas etapas pré-estimulação auditiva, quatro e oito meses após a doação de próteses auditivas não lineares.

\begin{tabular}{|c|c|c|c|c|c|c|c|}
\hline & Doação & & 04 meses & & 08 mese & & Total por \\
\hline \multirow{2}{*}{ Etapa } & Normal & Alterado & Normal & Alterado & Normal & Alterado & ba \\
\hline & $\%$ & $\%$ & $\%$ & $\%$ & $\%$ & $\%$ & $\%$ \\
\hline \multirow{2}{*}{ Total } & 0 & 31 & 18 & 13 & 24 & 7 & 31 \\
\hline & 0,0 & 100,0 & 58,1 & 41,9 & 77,4 & 22,6 & 100,0 \\
\hline
\end{tabular}

Legenda: Normal - número de idosos com resultado do ADAS-Cog considerado sem alteração cognitiva

Alterado - número de idosos com resultado do ADAS-Cog considerado sugestivo de alteração cognitiva

Total - número total de idosos participantes do estudo.

Fonte: Tabela elaborada pelas autoras.

Nota-se que a porcentagem de indivíduos com resultado normal aumenta no decorrer do tempo. As porcentagens de resultado alterado nas três ocasiões (doação, quatro e oito meses pós-estimulação auditiva) foram comparadas duas a duas obtendo-se os p-valores apresentados na Tabela 10. Se detectada diferença realizou-se o teste Comparações Múltiplas de Tukey.

Tabela 10 - p-valores obtidos na comparação entre as porcentagens de resultado alterado no ADAS-Cog nas ocasiões duas a duas.

\begin{tabular}{l|l|l}
\hline \multicolumn{3}{c}{ Comparação } \\
\hline Doação x 04 meses & Doação x 08 meses & $\mathbf{0 4}$ meses $\times 08$ meses \\
\hline$<0,001^{*}$ & $<0,001^{*}$ & 0,7455 \\
\hline
\end{tabular}

Legenda: Doação = início do uso de próteses auditivas não lineares.

04 meses = 04 meses de uso de próteses auditivas não lineares.

08 meses = 08 meses de uso de próteses auditivas não lineares.

$\left(^{\star}\right)$ = resultado estatisticamente significante

Fonte: Tabela elaborada pelas autoras.

Houve redução significante $(p<0,001)$ da frequência de ocorrência de resultado alterado no ADAS-Cog nas comparações doação x quatro meses e doação $x$ oito meses de uso de próteses auditivas evidenciando a melhora dos resultados sugestivos de alteração cognitiva após intervenção fonoaudiológica. 
Trata-se de estudo que objetivou investigar verificar os efeitos da estimulação acústica por meio do uso de próteses auditivas no desempenho cognitivo de idosos com avaliação sugestiva de alteração cognitiva por meio do teste ADAS-Cog, nas etapas pré, quatro e oito meses após intervenção fonoaudiológica por meio da adaptação de próteses auditivas. Sabe-se que indivíduos com perda auditiva podem apresentar pior desempenho nos testes verbais para avaliação cognitiva e que podem ser favorecidos com o uso de amplificação sonora (MIRANDA; DURANTE, 2009). Assim, faz-se necessária a estimulação acústica a fim de que se possam reduzir as dificuldades cognitivas (RAMOS; SIMÕES; ALBERT, 2001).

Quanto à idade, o grupo estudado apresentou média etária de 70,6 anos (Tabela 1) concordando com outros achados de estudos que avaliaram idosos (VAN HOOREN et al., 2005; GUERREIRO et al., 2006; MATTOS; VERAS, 2007; COX; XU, 2010; RUDNER; LUNNER; RÖNNBERG, 2011). Após análise estatística, este estudo mostrou que a idade não interferiu nos escores obtidos, corroborando com os resultados de outras pesquisas (BERTOLUCCI et al., 1994; CARVALHO; IORIO, 2009).

Quanto à escolaridade dos idosos participantes desta pesquisa observou-se que o grupo apresentou média de 4,9 anos de estudo (Tabela 2). Estes resultados são distintos dos achados do estudo de Guerreiro et al. (2006) no qual a escolaridade média foi de 9,3 anos. O achado da presente pesquisa justifica-se pelo fato de os idosos participantes pertencerem ao serviço público de saúde, no qual a maioria dos usuários apresenta uma escolaridade mais baixa quando comparada à dos idosos pertencentes ao serviço privado de saúde.

Ao agrupar as tarefas do teste ADAS-Cog nos domínios: memória (palavra evocada, orientação, reconhecimento de palavras, recordação/lembrança) - Tabela 3, linguagem (nomeação de objetos e dedos, comandos, linguagem falada, dificuldade na fala espontânea e compreensão) - Tabela 5 e praxia (praxia construtiva e praxia ideativa) - Tabela 7 observou-se uma diferença entre as etapas pré e pós-adaptação de próteses auditivas.

No domínio memória (palavra evocada, orientação, reconhecimento de palavras, recordação/lembrança), a redução na pontuação média foi de 1,89 pontos entre as etapas de doação e de quatro meses após o inicio das próteses auditivas; e, entre quatro e oito meses de uso de AASI, esta diferença foi de 1,61. Portanto, houve melhor desempenho dos idosos no domínio memória. Essa evolução foi gradativamente melhor com o maior tempo de uso das próteses auditivas. 
Em relação ao domínio linguagem (nomeação de objetos e dedos, comandos, linguagem falada, dificuldade na fala espontânea e compreensão) também pode-se perceber uma melhora nos escores em todas as avaliações. Entre a etapa de doação e a de reavaliação em quatro meses de uso de próteses auditivas esta diferença foi de 0,59 pontos; e entre quatro e oito meses a diferença foi de 0,92 pontos. Tais diferenças evidenciam um melhor desempenho dos idosos quanto ao domínio linguagem, principalmente entre as etapas de quatro e oito meses de uso de próteses auditivas, favorecendo a comunicação dos idosos no convívio social.

Observou-se que o domínio praxia (praxia construtiva e praxia ideativa) apresentou uma queda mais acentuada de 0,61 pontos entre as etapas de doação e quatro meses após início do uso das próteses auditivas. A diferença entre as etapas de doação e de oito meses foi de 0,39 pontos revelando assim, redução dos escores neste domínio, o que talvez possa ser explicado pela maior atenção dispensada às tarefas devido ao uso de amplificação sonora.

Assim, essas diferenças encontradas com o uso de próteses auditivas mostram que houve uma melhora do desempenho dos domínios cognitivos, o que evidencia que o uso de próteses auditivas pode proporcionar benefícios quanto aos aspectos cognitivos, corroborando com os dados obtidos na literatura pesquisada sobre o tema (LUZ et al., 2011).

O estudo do escore global do ADAS-Cog revelou que o número de idosos com resultados normal (sem alteração cognitiva) aumentou no decorrer da pesquisa, ou seja, o total de idosos sem alterações cognitivas passou de $0 \%$ (pré-intervenção audiológica) para 58,1\% em quatro meses e 77,4\% em oito meses pós-estimulação auditiva, evidenciando melhora no desempenho do teste. Tal fato pode ser justificado pelo maior contato com as informações de fala que os participantes passaram a ter após o início do uso de próteses auditivas reduzindo seu esforço em ouvir e liberando suas funções executivas para outras atividades mais relevantes naquele momento de escuta.

O presente estudo concorda com pesquisas de: (1) ACAR et al. (2011), na qual todos os 34 participantes apresentaram uma melhora significativa das condições cognitivas após três meses de uso de aparelhos de amplificação sonora individual (AASI); (2) LIN et al. (2011), apontando associação entre o grau da perda auditiva e as pontuações nos testes cognitivos, ou seja, quanto maior o grau de perda auditiva do idoso menor sua pontuação no teste Mini Exame do Estado Mental (MEEM). Mas, discorda dos achados de Hooren et al. (2005) que mostraram que o grupo de 56 idosos com próteses auditivas quando comparado ao grupo sem próteses auditivas apresentou melhora dos 
limiares auditivos com próteses auditivas após 12 meses de uso das mesmas, porém não houve melhora do desempenho cognitivo. Com base nos resultados obtidos pode-se inferir que o uso de amplificação sonora pelo idoso pode minimizar alterações cognitivas relacionas à memória, à linguagem e à praxia possibilitando, assim, melhor qualidade de vida no processo de envelhecimento.

\title{
5 Conclusão
}

Os idosos apresentaram melhora nos escores do teste Adas-Cog nos domínios cognitivos de memória, linguagem e praxia após a estimulação acústica por meio do uso de próteses auditivas.

\author{
COGNITIVE DOMAINS AND AUDITION: STUDY \\ OF ELDERLY NEW USERS OF HEARING AIDS
}

\section{abstract}

Objective: To verify the effects of acoustic stimulation through the use of hearing aids in the elderly with an evaluation suggestive of cognitive alteration. Methods: Thirty-one elderly patients with symmetric bilateral sensorineural hearing loss of moderate to severe severity with a score suggestive of cognitive impairment were evaluated, 15 male and 16 female, from 60 to 80 years old. These elderly patients were evaluated by the Alzheimer's Disease Assessment Scale-cognitive subscale (ADAS-Cog) test at the Health Audit Service of the University Hospital of the Escola Paulista de Medicina - UNIFESP - and were re-evaluated before, four and eight months after hearing aids fitting. The adaptation of hearing aids was performed according to the National Auditory Health Care Policy of the Federal Government - Brazil (SAS/MS \#432, November 14, 2000). Data analysis was performed using descriptive statistics, Variance Analysis (ANOVA) and Tukey multiple comparisons with a significance level of 5\%. Results: The 11 ADAS-Cog tasks were grouped into three cognitive domains: memory, praxis and language. The study showed that there was a significant gradient reduction $(p<0.001)$ in the scores throughout the evaluations, indicating improvement in the test responses. The percentage of elderly patients with cognitive alteration results increased from 100\% (pre-intervention stage) to $41.9 \%$ and $22.6 \%$, respectively, 
four and eight months after hearing AIDS fitting. Conclusion: The elderly showed improvement in ADAS-Cog responses after acoustic stimulation through the use of hearing aids.

keywords

Hearing Aids. Questionnaire. Cognition. Aged.

referências

ACAR, Baran; ALTINDAG, Muge Fethiye Yurekli; BABADEMEZ, Mehmet Ali; KARASEN Riza Murat. Effects of hearing aids on cognitive functions and depressive signs in elderly people. Archives of Gerontology and Geriatrics, Amsterdam, v. 52, n. 3, p. 250-252, May/June 2011.

ALBERT, Marilyn S.; HELLER, Hope S.; MILBERG, William. Changes in naming ability with age. Psychology and Aging, Arlington, VA, v. 3, n. 2, p. 173-178, June 1988.

ALLEN, N Harry; BURNS, Alistair; NEWTON, Valerie; HICKSON, Frances; RAMSDEN, Richard; ROGERS, Jenny; BUTLER, Sue; THISTLEWAITE, Gillian; MORRIS, Julie. The effects of improving hearing in dementia. Age and Ageing, Oxford, v. 32, n. 2, p. 189-193, 2003.

ALMEIDA, Mariana Ribeiro de; GUARINELLO, Ana Cristina. Reabilitação audiológica em pacientes idosos. Revista da Sociedade Brasileira de Fonoaudiologia, São Paulo, v. 14, n. 2, p. 247-255, 2009.

ARNETT, Peter A; RAO, Stephen M; GRAFMAN, Jordan; LOBECK, Lorri. Executive functions in multiple sclerosis: an analysis of temporal ordering, semantic encoding, and planning abilities. Neuropsychology, Washington, D.C., v. 11, n. 4, p. 535-544, Oct. 1997.

BARRETO, Sandhi Maria. Envelhecimento: prevenção e promoção da saúde. Cadernos de Saúde Pública, Rio de Janeiro, v. 22, n. 9, p. 2009, set. 2006.

BERTOLUCCI, Paulo Henrique Ferreira; BRUCKI, Sonia M D; CAMPACCI, Sandra R.; JULIANO, Yara. O Mini-Exame do Estado Mental em uma população geral: impacto da escolaridade. Arq Neuropsiquiatria, São Paulo, v. 52, n. 1, p. 1-7, mar. 1994.

BRASIL. Ministério do Planejamento, Orçamento e Gestão. Instituto Brasileiro de Geografia e Estatística. Perfil dos idosos responsáveis pelos domicillios no Brasil. Rio de Janeiro: IBGE, 2000.

CARVALHO, Laura Maria Araújo. Avaliação objetiva e subjetiva de idosos usuários de próteses auditivas e segundo o desempenho cognitivo e os sintomas depressivos. 2009. 107 f. Dissertação (Mestrado em Fonoaudiologia: Distúrbios da Comunicação Humana) - Universidade Federal de São Paulo, São Paulo, 2009.

COX, Robyn M; XU, Jingjing. Short and Long Compression Release Times: Speech Understanding, Real-World Preferences, and Association with Cognitive Ability. Am J Audiol. Reston, VA, v. 21, n. 2, p. 121-138, Feb. 2010.

DAVIS, Hallowell; SILVERMAN, Sol Richard. Auditory test hearing aids. In: Hearing and Deafness. New York: Holt, Rinehart and Winston, 1970. 
DOHERTY, Karen A; DESJARDINS, Jamie L. The benefit of amplification on auditory working memory function in middle-aged and young-older hearing impaired adults. Front Psychol, v. 6, p. 721, 2015. doi.org/10.3389/fpsyg.2015.00721.

FOONG, Jackie; ROZEWICZ, L; QUAGHEBEUR, Gerardine; RON, Maria. Executive function in multiple sclerosis: the role of frontal lobe pathology. Brain, London, v. 120, n. 1, p. 15-26, Jan. 1997.

GATES, George A; MILLS, John H. Presbycusis. Lancet, v. 366, p. 1111-1120, Sep. 2005.

GUERREIRO, Tania Cristina et al. Queixa de memória e disfunção objetiva de memória em idosos que ingressam na Oficina da Memóriaß na UnATI/UERJ. Revista Brasileira de Geriatria e Gerontologia, Rio de Janeiro, v. 9, n. 1, p. 7-20, abr. 2006.

INSTITUTO BRASILEIRO DE GEOGRAFIA E ESTATÍSTICA. Sinopse do Censo Demográfico de 2010. Rio de Janeiro: IBGE. Disponível em: <http://www.censo2010.ibge. gov.br/sinopse>. Acesso em: 21 out. 2012

KIESSLING, Jürgen; PICHORA-FULLER, Margaret Kathleen; GATEHOUSE, Stuart; WEDEL, $\mathrm{H}$ von. Candidature forand delivery of audiological services: Special needs of older people. Int J Audiol, 42(Supp 2), S92-S101, Aug. 2003

LIGHT, Leah L; SINGH, Asha. Implicit and explicit memory in young and older adults. $J$ Experimental Psychology: Learning, Memory, and Cognition. Washington, D.C., v. 13, n. 4, p. 531-541, Oct. 1987.

LIN, Frank R; FERRUCCI, Luigi; METTER, E Jeffrey; RESNICK, Susan M. Hearing Loss and Cognition in the Baltimore Longitudinal Study of Aging. Neuropsychology, Washington, D.C., v. 25, n. 6, p. 763-770, Nov. 2011

LUZ, Vivian Batista; SILVA, Monique Cantelli; SCHARLACH, Renata Coelho; IORIO, Maria Cecília Martinelli. Correlação entre as restrições de participação em atividades de vida diária e o benefício do uso de próteses auditivas em adultos e idosos. Revista da Sociedade Brasileira de Fonoaudiologia. São Paulo, v. 16, n. 2, p. 160-166, abr./jun. 2006.

MARQUES, Ana Cléia O; KOZLOWSKI, Lorena; MARQUES, Jair Mendes. Reabilitação auditiva no idoso. Revista Brasileira de Otorrinolaringologia. São Paulo, v. 70, n. 6, p. 806-811, nov./dez. 2004.

MIRANDA, Elisiane Crestani de; DURANTE, Alessandra Spada. Negative synergy: hearing loss and aging. Revista da Sociedade Brasileira de Fonoaudiologia. São Paulo, v. 14, n. 3, p. 435-436, 2009.

MULROW, Cynthia D; AGUILAR, Christine; ENDICOTT, James E; TULEY, Michael R; VELEZ, Ramon; CHARLIP, Walter S; RHODES, Mary C; HILL, Judith A; DENINO, Louis A. Quality-of-life changes and hearing impairment: A randomized trial. Ann. Intern. Med. v. 113, n. 3, p. 188-94, Sept. 1990. Doi: 10.7326/0003-4819-113-3-188.

NITRINI, Ricardo. Semiologia Neurológica. In: NITRINI, Ricardo; BACHESCHI, Luiz Alberto. A neurologia que todo médico deve saber. São Paulo, Santos, p. 19-22. 2. ed., 1991.

PICHORA-FULLER, Margaret Kathleen. Perceptual Effort and Apparent Cognitive Decline: Implications for Audiologic Rehabilitation. Seminars in Hearing, New York, v. 27, n. 4, p. 284-293, 2006

RAMOS, Luiz Roberto; SIMÕES, Eduardo J; ALBERT, Marilyn S. Dependence in activities of daily living and cognitive impairment strongly predicted mortality in older urban residents in Brazil: a 2-year follow-up. Am J Geriatrics Soc, v. 49, n. 9, p. 1168-1175, Sept. 2001.

RUDNER, Mary; RÖNNBERG, Jerker; LUNNER, Thomas. Working Memory Supports Listening in Noise for Persons with Hearing Impairment. Am J Acad Audiol, Reston, VA, v. 22, n. 3, p. 156-167, Mar. 2011. 
RUSSO, lêda Chaves Pacheco. Distúrbios da Audição: a presbiacusia. In: RUSSO, lêda Chaves Pacheco. Intervenção Fonoaudiológica na Terceira Idade. Rio de Janeiro: Revinter, p. 51-82, 1999.

RUSSO, lêda Chaves Pacheco; ALMEIDA, Kátia; FREIRE, Katya G. M. Seleção e adaptação da prótese auditiva para o idoso. In: ALMEIDA, Katia de; IORIO, Maria Cecília Martinelli. Próteses auditivas: fundamentos teóricos e aplicações clínicas. 2. ed. São Paulo: Lovise, 2003.

SANTIAGO, Livia Maria; NOVAES, Cristiane de Oliveira. Auto-avaliação da audição em idosos. Revista CEFAC, São Paulo, v. 11, supl. 1, p. 98-105, 2009.

SCHULTZ, Rodrigo Rizek; SIVIERO, Marilena Ochini; BERTOLUCCI, Paulo H F. The cognitive subscale of the "Alzheimer's Disease Assessment Scale" in a Brazilian sample. Braz J Med Biol Res, Ribeirão Preto, v. 34, n. 10, p. 1295-1302, Oct. 2001

VAN HOOREN, Susan A H; ANTEUNIS, Lucien J C; VALENTIJN, Susanne AM; BOSMA, Hans; PONDS, Rudolf; JOLLES, Jelle; VAN BOXTEL, Martin P J. Does cognitive function in older adults with hearing impairment improve by hearing aid use? Int $J$ Audiol, London, v. 44, n. 5, p. 265-271, May 2005.

WEINSTEIN, Barbara E.; AMSEL, Lynn. The relationship between dementia e hearing impairment in the institutionalized elderly. J Clin. Gerontol., v. 4, n. 3, p. 3-15, Feb. 1986. doi: 10.1300/J018v04n03_02.

Data de submissão: 11/11/2014

Data de aprovação: 26/07/2015 
